\title{
Integrated nuclear data utilization system for innovative reactors nuclear data processing and utilization system
}

\author{
N. Yamano ${ }^{1, a}$, J. Katakura ${ }^{2}$, K. Kato ${ }^{3}$, and M. Igashira ${ }^{1}$ \\ ${ }^{1}$ Research Laboratory for Nuclear Reactors, Tokyo Institute of Technology, Tokyo 152-8550, Japan \\ 2 Nuclear Data Evaluation Group, Japan Atomic Energy Agency, Tokai, Ibaraki 319-1195, Japan \\ 3 Division of Physics, Graduate School of Science, Hokkaido University, Sapporo, Hokkaido 060-0810, Japan
}

\begin{abstract}
A nuclear data processing and utilization system has been developed as a modular code system of the integrated nuclear data utilization system for innovative nuclear energy systems. The system was developed in part of the research project entitled "Fundamental Research and Development (R\&D) on Neutron Cross Sections for Innovative Reactors using Advanced Radiation Measurement Technology". The system is able to handle evaluated nuclear data in the ENDF-6 format to generate point-wise and group-wise cross sections in several formats, and has a capability to perform criticality and shielding benchmarks based on various criticality and shielding benchmark problems selected from database such as ICSBEP and SINBAD. The system is operated with a common graphical interface (CGI) in order to easily utilize the system through Internet for not only nuclear design engineers but also nuclear data evaluators. The system is expected to apply quality assurance program for the JENDL-4 project. An overview of the integrated nuclear data utilization system and the scheme of cross section generation and benchmark procedure in the system have been described as well as future development plan based on the system.
\end{abstract}

\section{Introduction}

An integrated nuclear data utilization system has been developed for innovative nuclear energy systems, which is part of the research project entitled "Fundamental Research and Development (R\&D) on Neutron Cross Sections for Innovative Reactors using Advanced Radiation Measurement Technology". [1]. The research project consists of development of advanced measurement technology, acquisition of neutron cross sections of minor actinides, and development of the integrated nuclear data utilization system to perform evaluation of nuclear data based on the cross section measurement in this project.

The system has been constructed as a modular code system which consists of a management system and two subsystems. The management system named CONDUCT controls system resource management of PC Linux cluster server and the user interface. A subsystem is the nuclear data search and plotting execution system named SPES [2]. Nuclear data such as EXFOR [3], JENDL-3.3 [4], ENDF/B-VI [5] and JEFF3.1 [6] can be searched and plotted in the subsystem. The other is the nuclear data processing and utilization subsystem which is able to handle JENDL-3.3, ENDF/B-VI and JEFF3.1 to generate point-wise and group-wise cross sections in several formats, and perform various criticality and shielding benchmarks for verification of nuclear data. The system is operated with a common graphical interface (CGI) in order to easily utilize the system through Internet for not only nuclear design engineers but also nuclear data evaluators. The architecture of the system is shown in figure 1 . The system flow of the integrated nuclear data utilization system is shown in figure 2 .

\footnotetext{
${ }^{a}$ Presenting author, e-mail: yamano@nr.titech.ac.jp
}

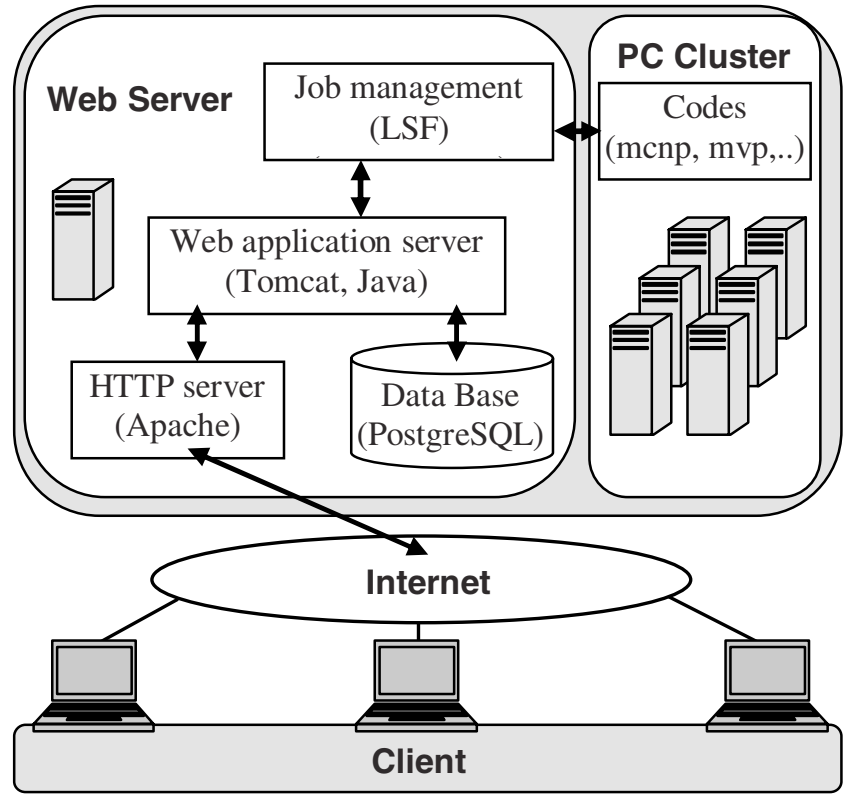

Fig. 1. Architecture of the integrated nuclear data utilization system.

\section{Integrated nuclear data utilization system}

\subsection{CONDUCT}

The management system named Consolidated Nuclear Data Utilization, Circulation and Transfer System (CONDUCT) controls system resource management of the PC Linux server and the user interface through Internet access. A web server in CONDUCT controls user authentication, requests on job execution, job status and data transfer. The background jobs requested by each user are executed in the PC cluster through 


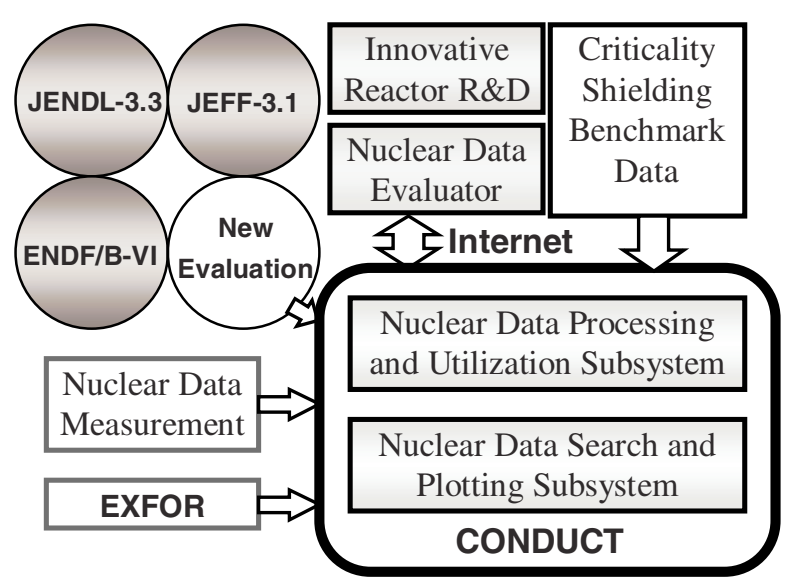

Fig. 2. Flow of the integrated nuclear data utilization system.

job management system named LSF. Common database such as indices of EXFOR and evaluated nuclear data libraries are controlled by using PostgreSQL.

\subsection{Nuclear data search and plotting subsystem, SPES}

The nuclear data search and plotting subsystem named SPES is used to search nuclear data such as experimental data including EXFOR and evaluated data in JENDL, ENDF/B and JEFF, and retrieve numerical data in tabular or figure format. The nuclear data newly measured and/or evaluated by user can be added and processed in this subsystem. The SPES is a powerful tool to compare experimental data such as EXFOR database and nuclear data in the ENDF-6 format. The SPES is designed for using through web browsers. The user can indicate arbitrary search conditions such as author, reference, year, projectile and target. If the search conditions agree with existing data, a data list will be displayed. If the user clicks the plot button on the display, the data will be displayed as shown in figure 3.

\subsection{Nuclear data processing and utilization subsystem, NUPS}

The nuclear data processing and utilization system named NUPS is able to handle JENDL-3.3, ENDF/B-VI and JEFF3.1 to generate point-wise and group-wise cross sections in several formats, and perform various criticality and shielding benchmarks for verification of nuclear data. Multi-group cross section covariance matrices are also generated in the COVERX format.

One of the features of the system is verification of nuclear data, by using various criticality and shielding benchmark problems selected from database such as International Criticality Safety Benchmark Experiments Project (ICSBEP) [7] and Shielding Integral Benchmark Archive and Database (SINBAD) [8]. The system is designed for users not only nuclear design engineers but also nuclear data evaluators to verify nuclear data for safety issues in the innovative reactor development.

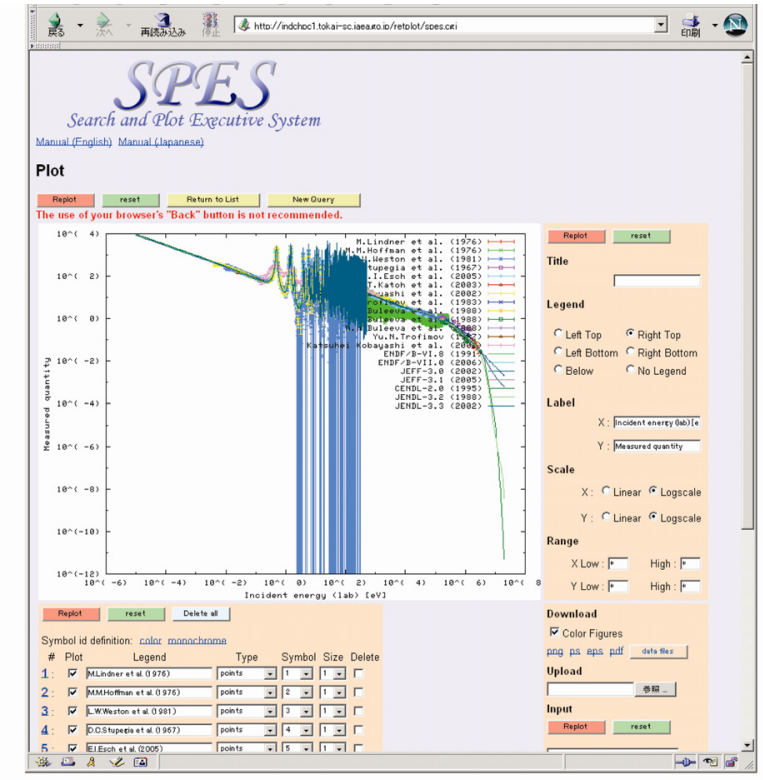

Fig. 3. A sample display using the nuclear data search and plotting subsystem, SPES.

Table 1. Specification of the nuclear data processing and utilization subsystem, NUPS.

\begin{tabular}{l}
\hline Nuclear Data: ENDF-6 format \\
\hline JENDL-3.3, ENDF/B-VI.8, JEFF-3.1 \\
\hline Library format: MCNP4C, MCNP5, MVP2, MATXS, COV- \\
ERX \\
\hline Processing code: NJOY99.125, LICEM, ERRORJ2.2 \\
\hline Processing parameter: energy group structure, temperature, \\
background cross section, processing tolerance, order of \\
Legendre coefficients, weighting spectrum, material com- \\
position, density, S( $\alpha, \beta)$, reaction type, photon production, \\
covariance \\
\hline Benchmark problem: suitable problems retrieved from ICS- \\
BEP, SINBAD database, etc. \\
\hline Criticality benchmark: PU, HEU, IEU, LEU, U233, MIX, \\
SPEC \\
\hline Shielding benchmark: O, Na, Al, Si, Ti, V, Cr, Fe, Co, Ni, \\
Cu, Zr, Nb, Mo, W, Hg SDT, ORNL, FNS, ASPIS, KfK, \\
OKTAVIAN, IPPE, NIST, JASPER \\
\hline
\end{tabular}

The system performance highly depends on the quality of benchmark problems so that appropriate benchmarks should be selected from existing database. A brief specification for cross section generation and benchmark problem is shown in table 1 . The cross section library formats are selected to be use of best-estimate codes.

The system mainly consists of two parts, one is the cross section generation suite, and the other is performing benchmarks. The main menu of the system is shown in figure 4 . If the cross section generation is selected, a menu for choosing the library format will appear as shown in figure 5. A pointwise library format or a group-wise format can be selected. If the user selects the point-wise format, a selection menu of MCNP [9] or MVP [10] format will appear as shown in figure 6. 


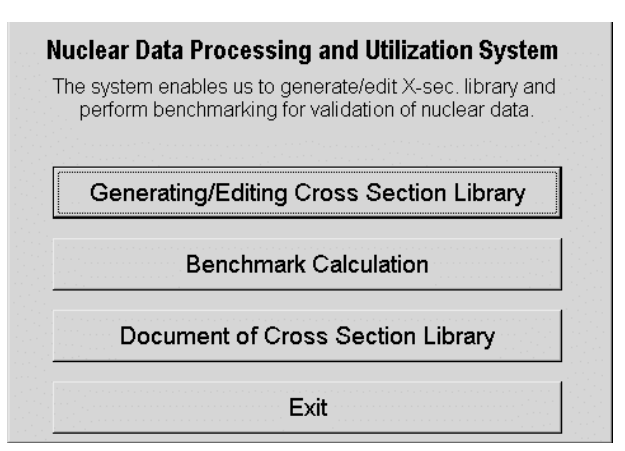

Fig. 4. Main menu of the nuclear data processing and utilization subsystem.

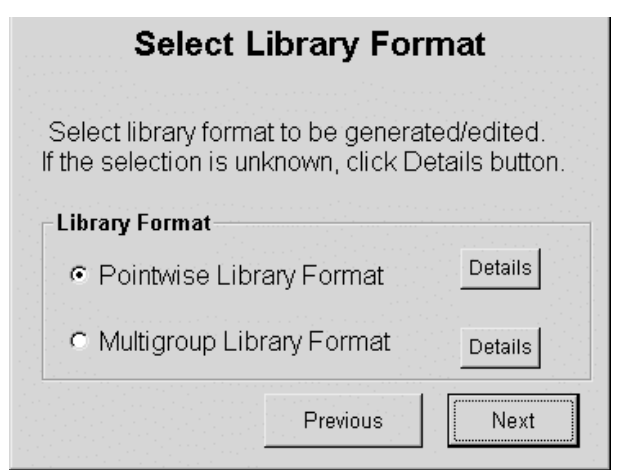

Fig. 5. A selection menu of cross section generation.

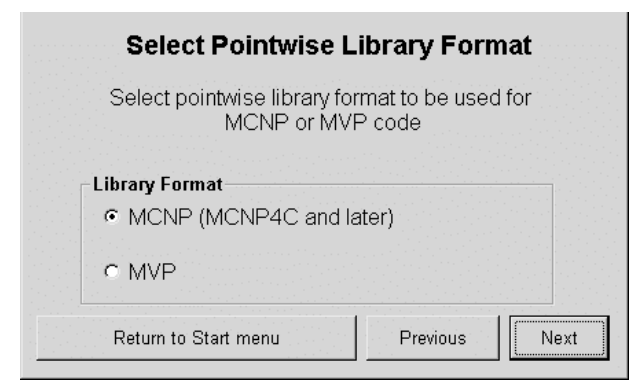

Fig. 6. A selection menu of point-wise cross section format.

If the user clicks the benchmark calculation button in the main menu, the following menu is displayed as shown in figure 7. In the selection menu, shielding or criticality benchmark can be selected. The criticality benchmark problems contained in the system are selected from ICSBEP benchmarks. The shielding benchmarks in the system are mainly adopted in the JENDL-3.3 verification.

If the criticality benchmark is selected in the menu of figure 7, the selection menu of ICSBEP will appear as shown in figure 8. Benchmark problems in ICSBEP are categorized by major items (PU, HEU, IEU, LEU, U233, MIX and SPEC), 4 physical forms and 4 spectrum types. The criticality benchmark problems can be selected from the hierarchy structure.

In the case of shielding benchmarks, problems are categorized by each element or nuclide. If the user selects element name such as iron, shielding benchmarks for iron available in

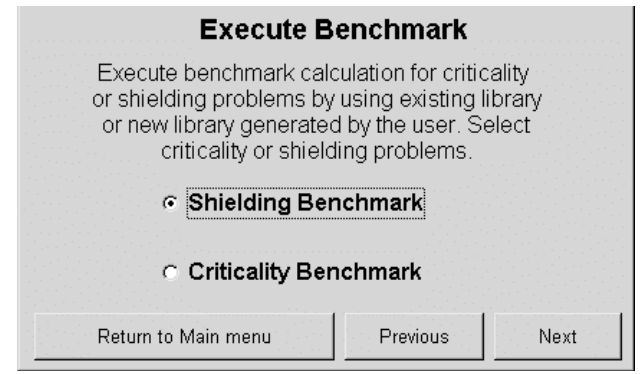

Fig. 7. A selection menu of benchmark calculation.

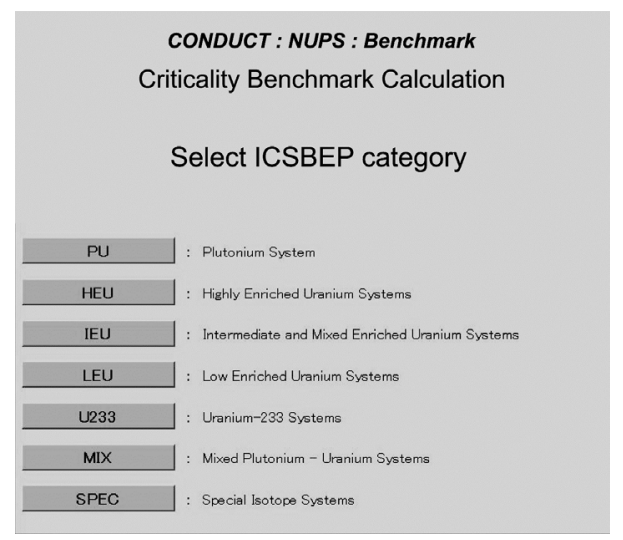

Fig. 8. A selection menu of ICSBEP benchmark category.

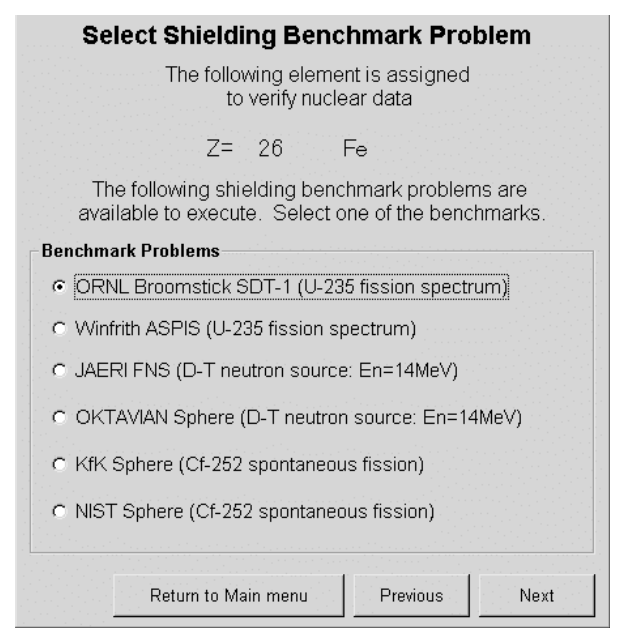

Fig. 9. A selection menu of shielding benchmark.

this system are displayed as shown in figure 9, so that the users can select suitable benchmark for their purpose.

The benchmark calculation is usually time consuming, so that the system puts the execution into a background process of the PC cluster and indicates the process identification number to the user. If the background process is completed, the information is notified to the user by e-mail, so that user can confirm the benchmark result.

If the user clicks the confirmation button in the benchmark menu, results will be displayed as shown in figures 10 and 11 for criticality and shielding benchmarks, respectively. In a sample case of criticality benchmark shown in figure 10, 


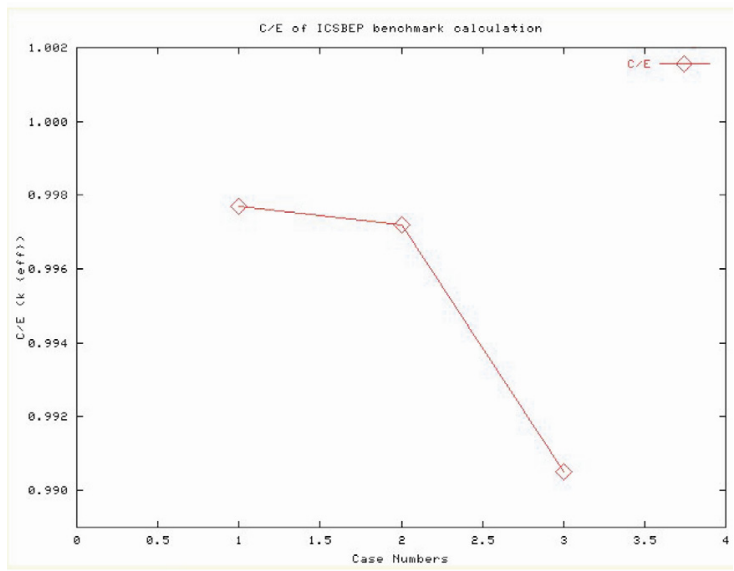

Fig. 10. A sample C/E plot for criticality benchmarks.

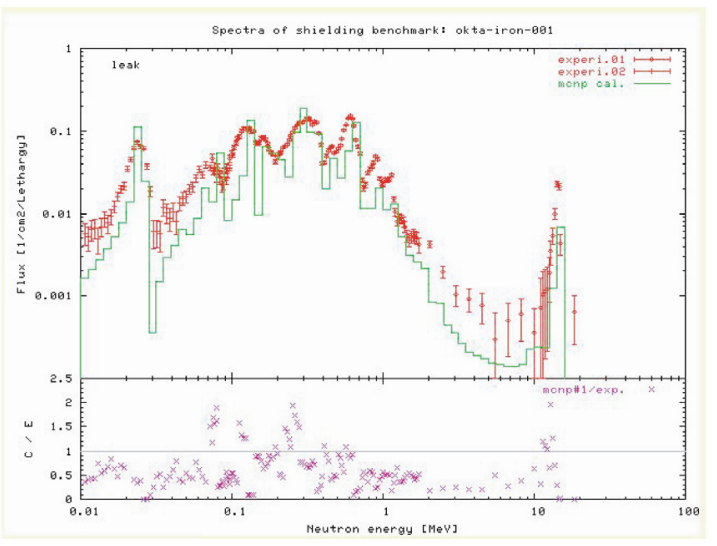

Fig. 11. A sample $\mathrm{C} / \mathrm{E}$ plot for shielding benchmarks.

the $\mathrm{C} / \mathrm{E}$ plot for three benchmarks is displayed as well as numerical results. In the case of shielding benchmark in figure 11 , the $\mathrm{C} / \mathrm{E}$ plot is also displayed as well as the energy spectra.

\section{Conclusion}

The nuclear data processing and utilization system has been developed as a module of the integrated nuclear data utilization system. The system is expected as a verification tool of nuclear data for development of innovative nuclear reactors because cross section generation from nuclear data and the various benchmarks for criticality and shielding problems can be easily performed. The system is now considering extension performing sensitivity analysis based on the covariance matrices generated in the system through the other nuclear data project. The system is expected to apply quality assurance program for the next JENDL project.

This work was performed under contract with Ministry of Education, Culture, Sports, Science and Technology (MEXT).

\section{References}

1. M. Igashira, M. Mizumoto, M. Oshima, A. Hasegawa, H. Harada, N. Yamano, H. Yamana, M. Baba, K. Kato, M. Sugawara, Y. Nagai, K. Kawade, A Nuclear Data Project in Japan, Proc. Int. Conf. on Nuclear Data for Science and Technology, Santa Fe, New Mexico, Sept. 26 - Oct. 1, AIP Conference Proceedings, Part 1, pp. 601-604 (2005).

2. N. Otuka, M. Aikawa, T. Suda, K. Naito, S. Korennov, K. Arai, H. Noto, A. Ohnishi, K. Kato, T. Nakagawa, T. Fukahori, J. Katakura, Web-Based Search and Plot System for Nuclear Reaction Data, Proc. Int. Conf. On Nuclear Data for Science and Technology, Santa Fe, New Mexico, Sept. 26 - Oct. 1, AIP Conference Proceedings, Part 1, pp. 561-564 (2005).

3. OECD/NEA Data Bank, http://www.nea.fr/html/dbdata/x4/ welcome.html

4. K. Shibata et al., Japanese Evaluated Nuclear Data Library Version 3 Revision-3: JENDL-3.3, J. Nucl. Sci. Technol. 39, 1125 (2002).

5. V. McLane (ed.), ENDF-102 Data Formats and Procedures for the Evaluated Nuclear Data File ENDF-6, BNL-NCS-4494501/04-Rev. (2001).

6. OECD/NEA Data Bank, The JEFF-3.0 Nuclear Data Library, JEFF Report 19 (2005).

7. A. Nouri, M.A. Thompson, J.B. Briggs, L. Scott et al., International Handbook of Evaluated Criticality Safety Benchmark Experiments, NEA/NSC/DOC(95)03 (2003).

8. H.T. Hunter et al., Shielding Integral Benchmark Archive Database, DLC-191 available from RSICC (2000).

9. J. Briesmeister (ed.), MCNP - A General Monte Carlo NParticle Transport Code, LA-12625-M (1993).

10. Y. Nagaya, K. Okumura, T. Mori, M. Nakagawa, MVP/GMVP II: General Purpose Monte Carlo Codes for Neutron and Photon Transport Calculations based on Continuous Energy and Multigroup Methods, JAERI 1348 (2004). 